Gallup, G. G., Jr., Montevecchi, W. A., \& Swanson, E. T. Motivational properties of mirror-image stimulation in the domestic chicken. The Psychological Record, 1972, 22, 193-199.

Hughes, R. N. Social facilitation of locomotion and exploration in rats. British Journal of Psychology, 1969, 60, 385-388.

Shashoua, V. E. Seasonal changes in the learning and activity patterns of goldfish. Science, 1973, 181, 572-574.
Tolman, C. W. Social facilitation of feeding behavior in the domestic chick. Animal Behaviour, 1964, 12, 245-251.

Welty, J D Experiments in group behavior of fishes. Physiological Zoology, 1934, 7, 85-128.

Zajonc, R. B. Social facilitation. Science, 1965, 149, 269-274.

\title{
On the establishment of a continuous repertoire*
}

\author{
SHEILA CHASE, ETHEL A. GELLER, and JEAN S. HENDRY \\ Hunter College of the City University of New York, New York, New York 10021
}

Pigeons trained to peck the response key directly below a spot of light which appeared in only three of five positions were tested with the spot in all five positions. Key choice, given these test stimuli, showed a mixture of functional control: on some trials, the key beneath the spot was chosen, indicating that the training produced a correspondence between novel points on the stimulus and response dimensions (a continuous repertoire); on other trials, the specific responses acquired during training were made.

Though behavior is often analyzed into discrete responses to discrete stimuli, much of it in fact consists of continuously variable activity related to continuous variations in stimulation. For example, to reach a ledge, a pigeon must adjust its flight pattern continuously in response to changing stimulation; similarly, to avoid a collision, a driver must adjust the position of the steering wheel in response to continuously changing stimulation. In situations of this sort, both stimuli and responses vary

*Reprints may be obtained from S. Chase, Department of Psychology, Hunter College, 695 Park Avenue, New York, New York 10021. along one or more continua; in the terminology of Skinner (1953), they comprise continuous fields. The functional control of behavior may be described by specifying the correspondence between points in the stimulus and response fields.

Some correspondences of this sort are surely the product of learning; however, the learning processes involved are very poorly understood. Since it is unreasonable to assume that all possible combinations of stimuli and responses have been encountered during prior learning experiences, some psychologists have assumed that the correspondence comes about through a process of extrapolation based on training during which 
a correspondence is established between a few isolated points or regions in the stimulus and response fields by differential reinforcement. This view is stated most explicitly by Skinner (1953). Referring to the act of reaching toward and touching a spot in the visual field, he says, "... all positions of the spot comprise a continuous field and all possible combinations of movements leading to contact form a corresponding field. The behavior is acquired upon specific occasions when specific responses toward specific locations are reinforced, but the organism almost inevitably acquires a coherent repertoire which can be described without referring to the punctuate origins of the two fields [pp. 116-117]."

Laboratory attempts to develop continuous repertoires by establishing a correspondence between two or more points in the stimulus and response fields have been quite unsuccessful. Wildemann and Holland (1972), for example, trained pigeons to peck on one end of a horizontal surface in the presence of a $1,250-\mathrm{Hz}$ tone and on the other end in the presence of a $2,500-\mathrm{Hz}$ tone. When tested with three tones intermediate in frequency, the pigeons continued to peck on one or the other end and showed no increase in the tendency to peck intermediate regions. In a further experiment, pigeons were trained to peck at three positions on the surface in the presence of three different tonal frequencies. Again, when they were tested with other frequencies, they confined their pecks to the three regions they had been trained on.

It has been suggested that the failure to obtain continuous repertoires may be due to the extinction of responding to intermediate regions. There are other possibilities, however. The reaching and touching situation described by Skinner differs in subtle ways from the situation studied by Wildemann and Holland. For one thing, the position of the spot serves not simply as a cue for an arbitrary response, but is intimately involved in the guidance of the response. In the hope of finding a situation that does yield correspondence between the stimulus and response continua, we arranged an experimental situation very similar to that described by Skinner.

\section{METHOD}

Two pigeons, maintained at $80 \%-85 \%$ of their free-feeding weight, were trained in a chamber that contained five pecking keys spaced $1.7 \mathrm{~cm}$ apart in a horizontal row, $14.2 \mathrm{~cm}$ above the floor of the chamber; $2.7 \mathrm{~cm}$ above the keys was a narrow horizontal screen $(2 \times 21 \mathrm{~cm})$, upon which a circular 1-cm-diam spot of light could be projected so as to appear directly above any one of the response keys. The spot positions and the corresponding keys are referred to as 1 to 5 , in order from left to right. A pilot light above the center of the display panel illuminated the chamber.

During the preliminary keypeck training, no spots were projected, and on a given trial, a single key was illuminated with green light. Pecks on this key were followed by reinforcement, a 2 -sec period of access to a tray of mixed grain. Both birds were first trained to peck Key 3 and were then given 10 reinforcements for pecks on each of the five keys, which were illuminated in haphazard order.

During subsequent discrimination training, all five response keys were illuminated on every trial. The spot appeared only above Keys 1, 3, or 5. A peck, during the trial, on the key below the spot was defined as correct. Immediately following a peck on any key, all keys were darkened for $7.5 \mathrm{sec}$, with the spot remaining on the display panel for the first $2 \mathrm{sec}$ of this intertrial interval. The spot position on the next trial was randomly determined, with the restriction that the spot appear in each of the three positions with approximately equal frequency. However, during the first 25 sessions, the spot position was not changed after trials on which an error was made. Sessions 1-27 each ended after 40 correct responses, with all correct responses followed by reinforcement. During the remaining five sessions, the percentage of correct responses reinforced was reduced to 50 , and the length of the session extended to 80 trials.

There followed a single test session during which the training stimuli (Spot Positions 1, 3, and 5) were presented along with two novel stimuli (Spot Positions 2 and 4). The stimulus order was randomized within blocks of five, and each stimulus was presented 16 times. Reinforcement was available following $50 \%$ of the correct responses to the training stimuli; in no instance did it follow a response to either of the novel stimuli.

\section{RESULTS AND DISCUSSION}

Figure 1 shows the proportion of responses to each of the five keys $\left(R_{1}\right.$ through $\left.R_{5}\right)$ as a function of spot position $\left(S_{1}\right.$ through $\left.S_{5}\right)$.

During the first 4 days of training (upper panel), the responses of both birds were divided approximately equally among the five keys. There is little correspondence between the key chosen and the position of the spot. However, during the last 4 days of training (middle panel), both birds chose the correct key, the one beneath the spot, on most trials.

The lowest panel shows the results of the test. The birds' behavior, given spots in Positions 1, 3, and 5, is comparable to that seen during the last 4 days of training. Of primary interest, of course, is the relation of their response pattern to the novel spot positions, 2 and 4. Note first that the pigeons pecked Keys 2 and 4 quite often during the test, whereas they rarely pecked these keys during the last 4 days of training. Virtually all the additional responses to Keys 2 and 4 occurred on trials on which the spot was directly above one of these keys; in fact, Bird 1 never pecked either of these keys unless the spot was directly above it. In other words, considering only those trials on which either Key 2 or Key 4 was pecked, we find that these keys were pecked quite often when the spot was immediately above them, and were pecked rarely or never when the spot was elsewhere. This feature of the results indicates that the training procedure did produce a correspondence between novel points on the stimulus and response continua. The birds appear to have learned the rule "peck the key below the spot."

On the other hand, on many occasions when the spot was shown in Position 2 or Position 4, the birds did not peck the key immediately below, but pecked one of the adjacent keys instead. This is the behavior expected if 


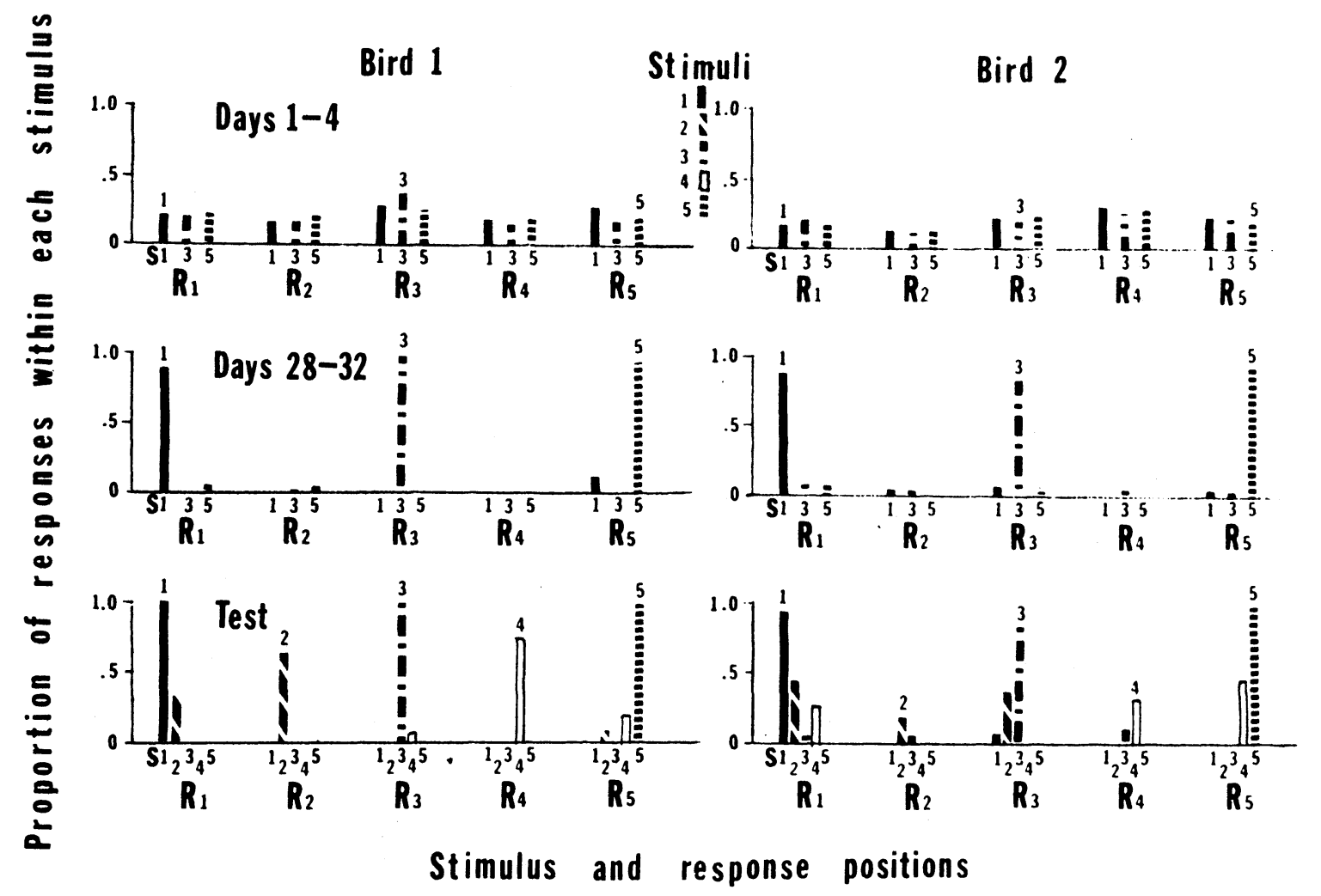

Fig. 1. Proportion of trials on which each of the five keys was pecked in the presence of each of the five stimuli (spot positions). The small numbers along the $X$-axis refer to the stimuli $\left(S_{1}\right.$ to $\left.S_{5}\right)$; the large numbers refer to the key choice $\left(R_{1}\right.$ to $R_{5}$ ). Bars with numbers above them represent the proportion of responses to the key directly below the spot.

the training procedure only established an association between the specific stimuli used and the specific responses reinforced in their presence. The pattern of responding to the novel stimuli follows from well-known principles of stimulus generalization. According to these principles, a novel stimulus that is similar to those used in training will evoke one of the specific learned responses. The probability with which the novel stimulus evokes each of the specific responses depends on its relative similarity to each of the training stimuli.

Thus, it seems that the behavior observed during the test reflects a mixture of two types of functional control. On some trials, the birds behaved in accordance with the rule "peck below the spot," whereas on other trials, they chose one of the specific responses acquired during training, the choice being governed by the similarity of the novel stimulus to each of the training stimuli.

The difference in the outcome of our experiment, which indicates that the procedures specified by Skinner do yield continuous repertoires, and others which show that they do not, undoubtedly results from differences in the stimulus continua studied. Our training situation is so arranged that just before a reinforced response is made, the spot is always located near the center of the pigeon's visual field, and just before an unreinforced response is made, the spot is in the periphery of the visual field or perhaps not seen at all. The distance of the spot from the center of the visual field may function as an error signal that the pigeons learn to minimize under the action of differential reinforcement. Observation of the pigeons' behavior suggests such a process. After they were well trained, each pige on typically moved his head horizontally until it was lined up with a point just above the spot, and then lowered it to peck the key. This sort of interaction between the discriminative stimuli and the topography of the response does not exist when the discriminative stimuli are diffuse sounds, as in the experiment of Wildemann and Holland (1972). Such interactions may well play a critical role in the development of continuous repertoires of behavior.

The finding that organisms when presented with novel stimuli tend to continue to make previously reinforced responses, perhaps in new combinations, does not mean that only specific associations can be formed. Training procedures very similar to those which previously produced only specific associations were shown here to produce continuous repertoires as well.

\section{REFERENCES}

Skinner, B. F. Science and human behavior. New York: Macmillan, 1953. Pp. 116-119.

Wildermann, D. G., \& Holland, J. G. Control of a continuous response dimension by a continuous stimulus dimension. Journal of the Experimental Analy sis of Behavior, 1972, 18, 419-434.

(Received for publication March 25, 1974.) 